\title{
Application of two-dimensional electrophoresis and mass spectrometry to screen endometriosis-related proteins
}

\author{
HANIKEZI TUERXUN $^{1 *}$, YANMEI ZHANG $^{2^{*}}$, FEI JI $^{1}$, AIXINGZI AILI $^{3}$, XINHUA YANG $^{1}$ and YAN DING ${ }^{1}$ \\ ${ }^{1}$ Gynecology Department, The First Affiliated Hospital of Xinjiang Medical University, Urumqi, Xinjiang 830054; \\ ${ }^{2}$ Department of Obstetrics, Xinjiang Uygur Autonomous Region People's Hospital, Urumqi, Xinjiang 830001; \\ ${ }^{3}$ Department of Gynecology, Shanghai Dongfang Hospital, Shanghai 200120, P.R. China
}

Received April 19, 2013; Accepted January 14, 2014

DOI: $10.3892 / \mathrm{mmr} .2014 .2142$

\begin{abstract}
The aim of the present study was to detect the differences in the protein expression between Uyghur females with or without endometriosis (EM). The two dimensional (2D) gel images of proteins extracted from the serum of Uyghur and Han females with EM and the controls were analyzed using Phoretix 2D software, and the differentially expressed proteins were identified primarily by database query. Having compared the reproducible $2 \mathrm{D}$ gel images of proteins from the serum of Uihgur and Han females with and without EM, 13 differentially expressed proteins were obtained from Uyghur females and eight differentially expressed proteins were obtained from Han females. The present study demonstrated ethnic differences in gene and protein expression between Uyghur and Han females with EM.
\end{abstract}

\section{Introduction}

Endometriosis (EM) is one of the most common gynecological diseases. It is a benign disease with malignant invasion and metastasis, and is linked with dysmenorrhea and infertility, with an incidence of $\sim 5-15 \%$. Although there has been almost a century of investigation, the cause of EM remains unclear. Previous studies have observed that EM may be caused by the interaction of a multifactorial-induced polygenic disease (1). Scholars have suggested the theory of 'eutopic endometrium determinism', that is the ectopic implantation of endometrial tissue depends on the differences in gene expression of the incumbent membrane. Comprehensive proteomics with the development of the Human Genome Project are capable of

Correspondence to: Dr Aixingzi Aili, Department of Gynecology, Shanghai Dongfang Hospital, No. 150 Jimo Road, Shanghai 200120, P.R. China

E-mail: aixingzicn@126.com

*Contributed equally

Key words: Uyghur, Han, endometriosis, immunoelectrophoresis, two-dimensional, protein processing the overall, dynamic, quantitative study of protein composition, function and correlation. The application of proteomics technology for EM is capable of generating the protein expression profile in patients with EM and revealing the pathogenic mechanisms at the molecular level, providing a non-invasive, more sensitive and specific diagnostic method. The focus of proteomics is to locate the expression of different proteins in disease conditions, which may become important tools for clinical diagnosis, particularly for early diagnosis. Ethnic differences exist in clinical medicine. Drug metabolism between the various ethnic groups in China are different. Therefore, the identification of different genes and proteins between the uterine EM patients of the Uygur and Han ethnicity may further guide the etiology, diagnosis and clinical treatment of EM.

\section{Subjects and methods}

Patients. All cases of EM were confirmed by laparotomy or laparoscopy at The First Teaching Hospital of Xinjiang Medical University (Urumqi, China). The ectopic endometrium and eutopic endometrium were obtained during surgery. EM lesions were obtained by biopsy. EM cases were included according to the following criteria: All cases had chocolate cysts of the ovaries and were obtained from Uyghur and Han patients who were native of different regions of the Xinjiang Uyghur Autonomous Region, were between the age of 18 and 45 years, had a regular menstrual cycle (28-32 days), exhibited no other endocrine, immune or metabolic diseases and did not use hormones preoperatively for three months. All specimens of endometrial and chocolate cysts were confirmed pathologically. There were a total of 10 samples of EM serum and eight samples of non-EM serum from Uygur patients, and 10 samples of EM serum and nine samples of non-EM serum from Han patients. Written informed consent was obtained from the patients and the study was approved by the Ethics Committee of Xinjiang Medical University.

Methods. The method of two dimensional (2D) polyacrylamide gel electrophoresis (PAGE) for protein expression profile analysis is described thoroughly in 'Proteomics' (2). Table I presents the reagents and the volumes used in each tube in 2D PAGE. The methods used are described below. 
Removal of plasma/serum albumin and $\mathrm{Ig} G$. Tris- $\mathrm{HCl}$ solution (10 mM; serum binding buffer, $\mathrm{pH}$ 7.4; Beijing Baier Tepp Biological Technology Development Co., Ltd., Beijing, China) was used to equilibrate the column filling, followed by centrifugation $(2,000 \mathrm{x} \mathrm{g} ; 20 \mathrm{sec})$. Then the solution in the column filling was removed. Dried $(0.02 \mathrm{~g})$ column filling was added to the Eppendorf tube. Plasma/serum $(25 \mu 1 ; 1: 3)$ was diluted with $10 \mathrm{mM}$ Tris- $\mathrm{HCl}$ solution $(\mathrm{pH} 7.4)$ and added to the Eppendorf tube, followed by oscillation for $40 \mathrm{~min}$. Following centrifugation $(2,000 \mathrm{x} \mathrm{g} ; 20 \mathrm{~min})$, the supernatant was collected. Tris- $\mathrm{HCl}$ solution $(\mathrm{pH} 7.4 ; 10 \mathrm{mM})$ was used to equilibrate protein $\mathrm{A}$, followed by centrifugation $(500 \mathrm{x} \mathrm{g}$; $1 \mathrm{~min})$. The redundant solution was then removed. Dried protein A $(0.04 \mathrm{~g})$ was added to the Eppendorf tube and the supernatant was added, followed by oscillation for $40 \mathrm{~min}$. Following centrifugation (500 x g; $5 \mathrm{~min}$ ), the supernatant was collected and the concentration of supernatant was measured by $2 \mathrm{D}$ electrophoresis.

\section{Determination of protein content using an improved Bradford method}

Basic principles. The protein was bound to the Coomassie brilliant blue G-250 dye and the maximum absorption peak of the dye was altered from 465 to $595 \mathrm{~nm}$. In a linear range, the reaction solution at $595 \mathrm{~nm}$ was proportional to the quantity of protein in the reaction. The increase in absorbance at $595 \mathrm{~nm}$ was determined so that the quantity of protein could be obtained.

Determination of the standard curve. The protein standard solution and melt diluent were removed and centrifuged at 1,200 x g for 3 min. Coomassie Brilliant Blue G-250 working solution (Sigma-Aldrich, St. Louis, MO, USA) was measured out, with $3.5 \mathrm{ml}$ for each tube. Hydrochloric $(\mathrm{HCl})$ acid $(0.1 \mathrm{~N})$ and double-distilled water $\left(\mathrm{ddH}_{2} \mathrm{O}\right)$ were prepared. The nine dry and clean test tubes were numbered and the reagents are shown in Table I. The test tubes were agitated at room temperature for $30 \mathrm{sec}$ following the addition of $3.5 \mathrm{ml}$ Coomassie Brilliant Blue G-250 to each tube.

Determination of sample. In a dry and clean test tube, $80 \mu \mathrm{ldd} \mathrm{H}_{2} \mathrm{O}$ was subsequently added to the dilution buffer, which was adjusted to the measuring range of the protein concentration of the sample $(10 \mu \mathrm{l})$, adding $0.1 \mathrm{~N} \mathrm{HCl}(10 \mu \mathrm{l})$, $30 \mathrm{sec}$ after $3.5 \mathrm{ml}$ Coomassie Brilliant Blue G-250 was added.

Sample protein content. The protein content of the original sample $(\mathrm{mg} / \mathrm{ml})=$ dilution factor $\mathrm{x}$ predicted value $(\mu \mathrm{g}) / 10 \mu \mathrm{l}$.

Instructions for IPGphor isoelectric focusing system. The strip holders with the desired length and number were thoroughly cleaned and dried using a soft bristle toothbrush and immobiline DryStrip rehydration solution (Xing-Yi Biotechnologies, Inc., Taiwan, China). The swelling liquid and sample were prepared. The strip length was determined according to the selected sample $(7 \mathrm{~cm}, \sim 125 \mu \mathrm{l} ; 13 \mathrm{~cm}$, $\sim 250 \mu \mathrm{l} ; 18 \mathrm{~cm}, \sim 340 \mu \mathrm{l} ; 24 \mathrm{~cm}, \sim 450 \mu \mathrm{l})$ and the extent of liquid bubble expansion was determined. According to the sample size, the required sample volume plus the quantity of swelling liquid were calculated and were thoroughly mixed. The sample solution was added dropwise to the strip holder evenly. The desired length was subsequently removed and the $\mathrm{pH}$ range was determined using IPG strips (Amersham
Pharmacia Biotech Inc., Piscataway, NJ, USA), according to the manufacturer's instructions.

PAGE and silver staining. The polyacrylamide gel was prepared (acrylamide from Sigma-Aldrich) and attached to the glass plate for electrophoresis. The pre-electrophoresis was performed and the sample was added, followed by electrophoresis at a constant power of $2.5 \mathrm{~W}$ for $45 \mathrm{~min}$ and $15 \mathrm{~W}$ for 5-7 h. Following electrophoresis, the gel was removed and subsequent to fixation, silver staining was performed for 20 min (0.625 g AgNO3; $100 \mu 137 \%$ formaldehyde), followed by water washing and coloration.

Database retrieval. The present study was designed to determine the isoelectric point and molecular weight of differentially expressed proteins in order to add them to the website http:// www.expasy.org. The software TagI-dent (Image Master 4.01; Amersham Pharmacia Biotech Inc.) was used to retrieve the Swiss-Prot database, looking for the protein matched to these two parameters. The range for the isoelectric point varied by 0.1 and the molecular weight ranged between 0.5 and $5 \%$.

Matrix-assisted laser desorption/ionization time-of-flight mass spectrometer (MALDI-TOF-MS) analysis. The candidate protein spots on the $2 \mathrm{D}$ electrophoresis gel were stained and numbered. The selected spots were cut along the edge with a razor blade and placed in a sample tube containing $50 \%$ (by volume) acetonitrile (Sigma-Aldrich) and $25 \mathrm{mmol} / 1$ ammonium bicarbonate (Rianlon Chemical Experiment Plant, Tianjin, China) solution for decoloration and were subsequently subjected to vacuum centrifugation for desiccation. Trypsin was prepared with $25 \mathrm{mmol} / \mathrm{l}$ ammonium bicarbonate solution to give a final concentration of $0.01 \mathrm{~g} / \mathrm{l}$. Then $8-10 \mu \mathrm{l}$ trypsin was added to each sample tube and insulated at $37^{\circ} \mathrm{C}$ for $20 \mathrm{~h}$. Trifluoroacetic acid (120 $\mu \mathrm{l}$ of 5\% by mass) was added to each sample (Promega Corporation, Madison, WI, USA) and was subsequently incubated at $40^{\circ} \mathrm{C}$ for $1 \mathrm{~h}$, and the supernatant was aspirated. To this, $2.5 \%$ trifluoroacetic acid and $120 \mu \mathrm{l}$ of $50 \%$ acetonitrile solution were added and subsequently incubated at $30^{\circ} \mathrm{C}$ for $1 \mathrm{~h}$. The supernatants were combined, freeze-dried and dissolved with $10 \mu \mathrm{l}$ of $0.5 \%$ trifluoroacetic acid. Of this, $1 \mu 1$ was mixed with a saturated $\alpha$-cyano-4-hydroxy cinnamic acid (Promega Corporation) solution of trifluoroacetic acid and $50 \%$ acetonitrile, which was dried at room temperature. The peptide mass fingerprint was obtained from reflex tests using a mass spectrometer with a laser wavelength of $337 \mathrm{~nm}$.

\section{Results}

Protein patternanalysis by 2 Del electrophoresis. The samples from the Uyghur and Han Chinese patients were assessed three times. The results demonstrated that three profiles in the dimensional electrophoresis were particularly similar in each group. The average number of protein spots was $965 \pm 52$ and $1024 \pm 72$, respectively. The protein glue diagrams for Uyghur EM patients and the control group are shown in Fig. 1A and B. The protein glue diagrams for the Han EM patients and the control group are shown in Fig. 1C and D. Phoretix 2D software was applied to analyze the differentially expressed 
Table I. Relative absorbance in each tube at $595 \mathrm{~nm}$.

Tube number

\begin{tabular}{lrrrrrrrrr}
\cline { 2 - 9 } Solution $(\mu \mathrm{l})$ & 0 & 1 & 2 & 3 & 4 & 5 & 6 & 7 & 8 \\
\hline $\mathrm{ddH}_{2} \mathrm{O}$ & 80 & 80 & 80 & 80 & 80 & 80 & 80 & 80 & 80 \\
Protein standard solution & 0 & 1 & 2 & 3 & 4 & 5 & 6 & 8 & 10 \\
Diluent & 10 & 9 & 8 & 7 & 6 & 5 & 4 & 2 & 0 \\
$0.1 \mathrm{~N} \mathrm{HCl}$ & 10 & 10 & 10 & 10 & 10 & 10 & 10 & 10 & 10 \\
\hline
\end{tabular}

$\mathrm{ddH}_{2} \mathrm{O}$, double-distilled water.

protein. The proteins with expression levels that were more than three times higher than that of the control group and were observed in at least two pairs of samples with the same change, were considered to be the different protein spots.

Determination of differentially expressed proteins through database retrieval. In the Uygur group, there were eight differentially expressed protein spots in the serum, while there was no expression in the normal controls (Table II). The distribution of the peak values of the differential proteins are shown in Fig. 2A. In the Han group, there were 13 differentially expressed protein spots in the serum, with no expression in normal controls (Table III). The distribution of the peak values of differential proteins is shown in Fig. 2B.

\section{Discussion}

The alteration in protein structure or quantity may lead to the occurrence of EM (2). By comparing proteins in samples of disease and normal tissue, different proteins may be identified, and those that correspond to the specific disease may be used as markers of the disease or a target-molecule for a drug. Due to individual differences in steroid hormones and the menstrual cycle, the single protein or small group of protein is particularly difficult to conclusively confirm. Previously, Rout et al (3) applied SDS-PAGE technology to compare the serum and peritoneal fluid between EM patients and a control group, to obtain evidence of the impact of reproductive ability in EM patients and the autoimmune system. The results demonstrated that there was no significant difference in the protein spectrum in the serum and peritoneal fluid between the two groups. However, in 18 of 20 patients with secretion of peritoneal fluid, the authors identified a protein that had not been described previously (molecular weight: $70,000 \mathrm{kDa}$ ), which did not appear in the proliferative phase samples. Numerous animal models have been used in EM-related research, including baboons, rhesus monkeys, rats and mice. However, certain species do not have a menstrual cycle and only primates suffer from EM in the wild. Newman et al (4) applied the animal model of surgeryinduced EM in rats and observed that ectopically implanted endometrium and eutopic endometrium may produce different proteins according to the SDS-PAGE method. These two proteins were termed ENDO-1 (molecular weight range, 40-50 $\mathrm{kDa}$; isoelectric point, 4.0-5.2) and ENDO-2 (molecular weight range, $28-32 \mathrm{kDa}$; isoelectric point, 7.5-9.0). Certain studies have demonstrated that the haptoglobin from EM lesions may combine with macrophages, thereby reducing its adhesion ability. Therefore, this may account for the pathogenesis of EM (5-7). In the serum and peritoneal fluid of EM patients, the concentration of tissue inhibitor of matrixmetallopeptidase inhibitor 1 (TIMP-1) was significantly lower than that observed in the controls. Therefore, the abnormal expression of TIMP-1 may contribute to the incidence of EM and may be a potential marker for the diagnosis of the disease. Nisolle et al (7) obtained the peritoneal fluid from infertile women who had no EM and normal controls in order to process the proteomic analysis with 2-DE technology and observed that the ENDO-1 protein existed in the peritoneal fluid of all patients. The molecular weight of ENDO-1 in rats was $40-50 \mathrm{kDa}$, while the molecular weight of human ENDO-1 was 32-40 kDa. No significant difference in the protein profiles was identified at different stages in the menstrual cycle according to data analysis. The range of ENDO-1 protein spot numbers was between 5 and 12 . When compared with the number of infertile women who had no EM (average no. 8) and the normal controls (average no. 7), the spot number of the EM patients (average no. 11) was higher. Wherein there was a protein with an isoelectric point of 5.8, and a molecular weight of $32 \mathrm{kDa}$ (termed EPF-32), which was elevated in the peritoneal fluid in 18 of the 19 EM patients, two of the seven patients in the infertility group and none of the patients in the control group. In the present study, the ENDO-2-like protein was not observed in the peritoneal fluid and whether EPF-32 is a diagnostic marker for EM was not verified. Küster et al (8) conducted a study of 24 female patients, who were divided into four groups: The mild EM group, the severe EM group, the simple infertility group and the normal control group; with six cases per group. The peritoneal fluid was analyzed with 2-DE proteome technology. The results revealed that the type and quantity of the proteins had significant differences. Demir et al (9) used the proteome analysis of peritoneal mesothelial cells during the human countercurrent blood induced transformation of epithelial cells to interstitial cells. It has been observed that countercurrent menstrual blood is capable of inducing the transformation of peritoneal mesothelial epithelial cells into mesenchymal cells, which may lead to cell contraction and the exposure of the extracellular matrix. Endometrial tissue initially adhered to the extracellular matrix and subsequently 
Table II. Proteins specifically expressed in Uyghur patients.

\begin{tabular}{ll}
\hline Site & \multicolumn{1}{c}{ Name and description of protein } \\
\hline 55 & gil40040316, (1) actin-like-7- $\alpha,(2) \gamma$-aminobutyric-acid receptor $\pi$ subunit \\
123 & gil56202824, (1) $14-3-3$ protein $\varsigma,(2)$ hepatoma-derived growth factor \\
20 & gil21040255, splicing factor, arginine/serine-rich 12 (Homo sapiens) \\
34 & gil7650400, immunoglobulin heavy chain variable region (Homo sapiens) \\
51 & gil33188435, deleted in liver cancer 1 isoform 3 (Homo sapiens) \\
48 & gil825615, $\alpha 2$-macroglobulin (Homo sapiens) \\
57 & gil89037979, predicted: hypothetical protein XP_938584 (Homo sapiens) \\
169 & gil3023686, excitatory amino acid transporter 5 (retinal glutamate transporter)
\end{tabular}

Table III. Proteins specifically expressed in Han patients.

\begin{tabular}{ll}
\hline Site & \multicolumn{1}{c}{ Name and description of protein } \\
\hline 62 & 55 for gil21439292, Unnamed protein product [Homo sapiens] \\
108 & 95 for gil40354192, Keratin 10 [Homo sapiens] \\
84 & 25 for gil52840231, MHC class II antigen [Homo sapiens] \\
83 & 49 for gil89027466, Predicted - similar to Eukaryotic translation initiation factor 3 subunit 2 (eIF3 p36) \\
27 & 74 for gil24659602, Keratin 16 [Homo sapiens] \\
26 & 41 for gil70798083, Immunoglobulin heavy chain variable region [Homo sapiens] \\
25 & 50 for gil83405621, Unknown (protein for IMAGE: 5585236) [Homo sapiens] \\
64 & 48 for gil21439292, Unnamed protein product [Homo sapiens] \\
151 & 50 for gil23272803, MGC40042 protein [Homo sapiens] \\
137 & 321 for Mixture 1, gil1147569 + gil401079 semenogelin I [Homo sapiens] and SPIN-2 \\
8 & 52 for gil39645832, C14orf10 protein [Homo sapiens] \\
7 & 49 for gil40538736, RAN binding protein 10 [Homo sapiens] \\
6 & 59 for gil57164405, TPR domain, ankyrin-repeat and coiled-coil containing 2 [Homo sapiens] \\
\hline
\end{tabular}

eIF-3 $\beta$, eukaryotic translation initiation factor-3 subunit 2; SPIN-2, spindlin-like protein 2; TPR, tetratricopeptide repeat; MHC, major histocompatibility complex.

adhered to the entire peritoneal cavity. The present study was designed to apply the radioisotope tracer and proteomics technology (2-DE and MALDI-TOF-MS) to detect the changes in protein expression and the phosphorylation of intermediate skin cells during the process of endometrial transformation. In total, 324 protein spots were detected and analyzed, 73 of which were identified, including 35 proteins with expression changes associated with the cytoskeleton signal transduction, the redox state and ATP generation, and four proteins with abnormal phosphorylation, including integrin-1 (actin junction protein and the substrate of tyrosine kinase receptor), tropomyosin- $\alpha$ (regulator of actin stability and cell morphology), elongation factor- $1 \delta$ and the $\beta$-chain of ATP synthase.

The spot no. 108 in Han uterine EM serum was type I cytoskeletal protein keratin 10 , which is capable of reducing the phosphorylation capacity of cells, thus affecting signaling, which may in turn impact cell proliferation. Miettinen et al (10) revealed that the expression of keratin increased in the hemangioma tissues. In keratin gene-transgenic mice, the epithelial cells in the pancreas may exhibit atypical hyperplasia, which is related to cancer. Biological pathway analysis identified that cytoskeletal remodeling via keratin intermediate filaments, processing of cystic fibrosis transmembrane receptor, the glucocorticoid receptor subunit $\alpha$ and heat shock factor 1 were all significantly over-represented features in EM patients. Spot no. 137 was spindle-like protein 2 (SPIN2), which was located in the nucleus and may promote cell proliferation and increase the ratio of cells in the G2/M phase cells. Consequently, it may be a tumor formation-related oncogene. Spot no. 84 was the major histocompatibility complex (MHC II antigens), which has a regulatory role in the immune system and translocates to the cell surface subsequent to binding to an antigen. It has been hypothesized that certain defects in MHC II class proteins cause mammals to be susceptible to diabetes. Gao et al (11) revealed that the MHC-I antigen on endometrial stromal cells may inhibit the toxicity of natural killer (NK) and NK-like $\mathrm{T}$ cells, while the level of MHC-I antigen may be adjusted. Cody et al (12) reported that ectopic endometrial tissue may express the MHC II antigen, thus presenting auto-antigens to the $\mathrm{T}$ and $\mathrm{B}$ cells and inducing endometrial antibodies. 
A

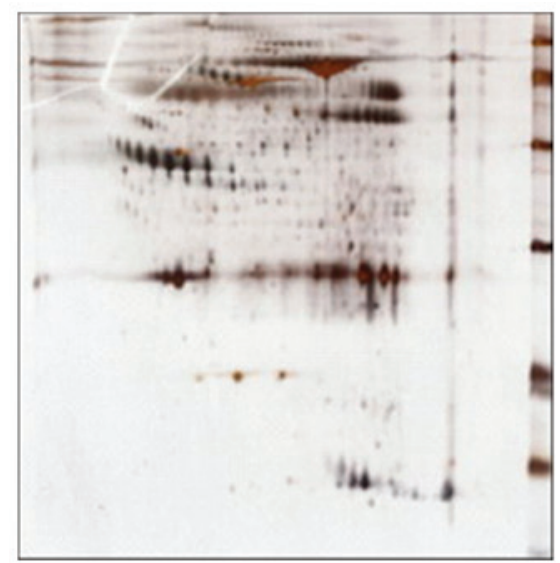

B

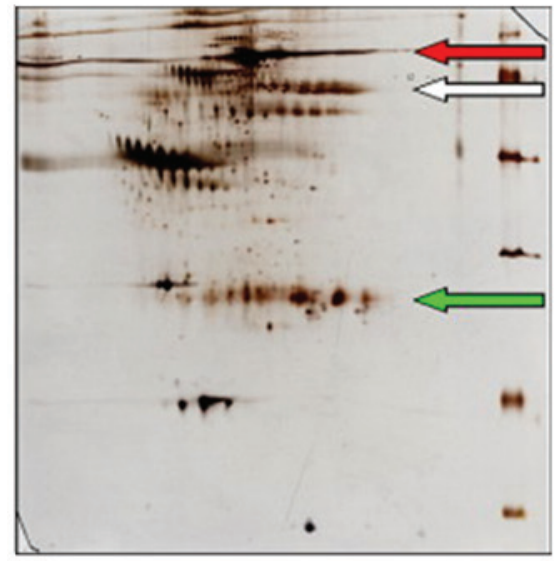

C

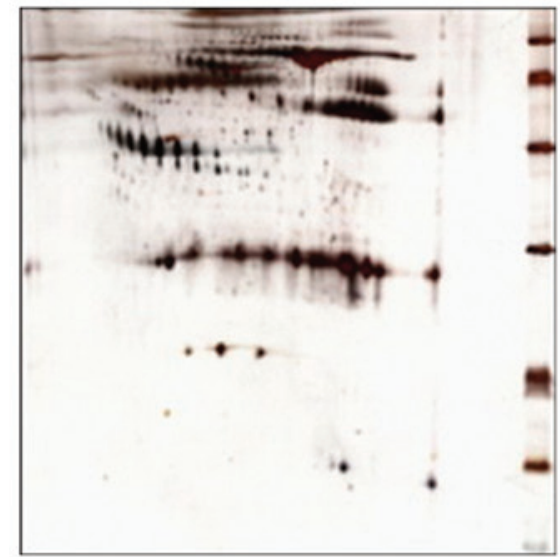

D

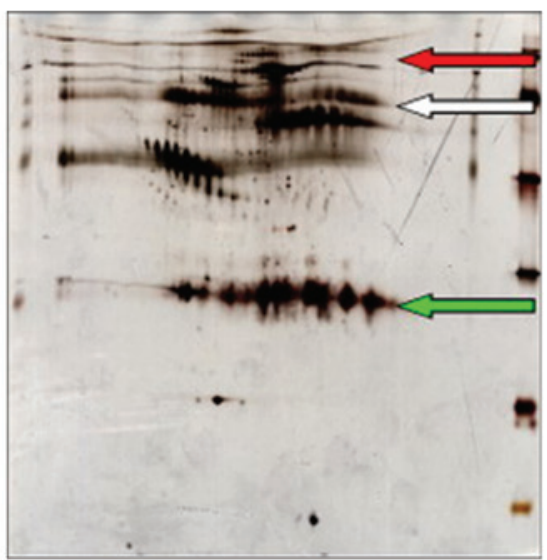

Figure 1. Protein glues of Uyghur and Han patients with EM. (A) Protein glues of Uyghur patients with EM in the disease group. (B) Protein glues of Uyghur patients with EM compared with the control group. (C) Protein glues of Han patients with EM in the disease group. (D) Protein glues of Han patients with EM compared with the control group. Red arrow, albumin; white arrow, IgG heavy chains; green arrow, low molecular weight protein. EM, endometriosis.
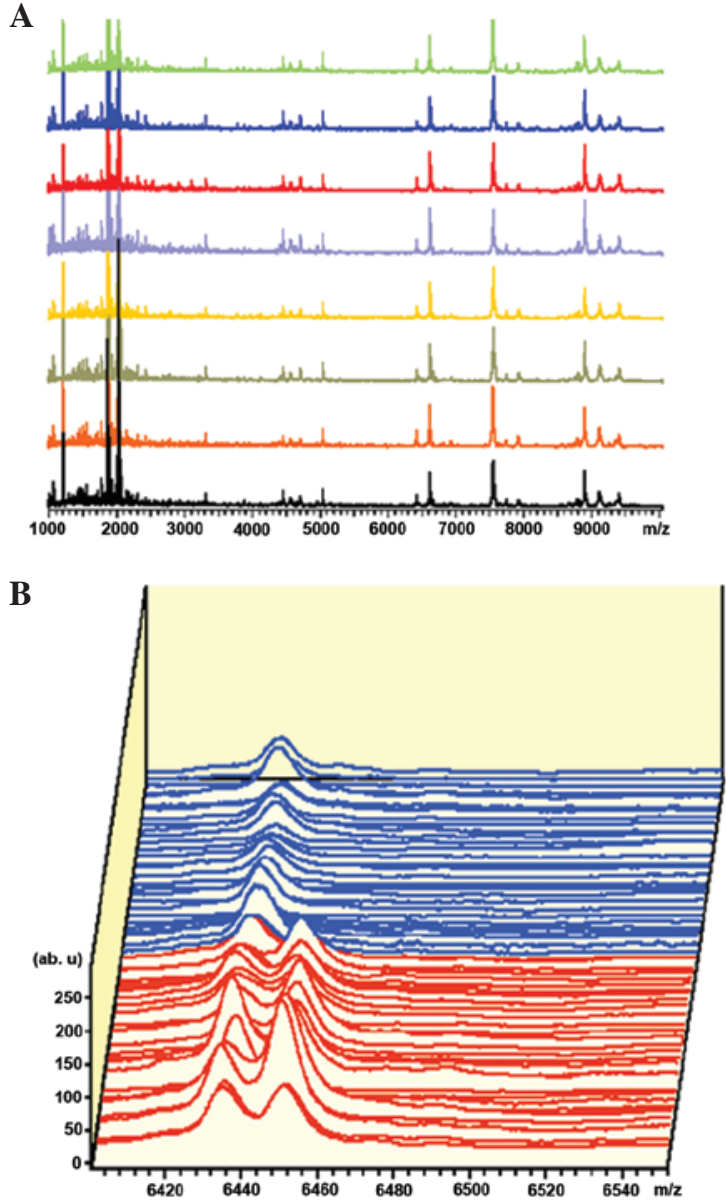

Figure 2. Peak value distribution of differential protein expression. (A) Peak value distribution of the differential proteins of Uyghur patients. (B) Peak value distribution of the differential proteins of Han patients with EM. Red, control group; blue, disease group. EM, endometriosis.

These autoantigens are primarily small endometrial proteins with molecular weights of 26, 34 and $42 \mathrm{kDa}$. The antigens combined with antibodies and were deposited in the uterus and ectopic lesions. The compound may activate the complement system and destroy the structure of the endometrium. Under the electron microscope, uterine cells exhibited a hypoplastic state, endometrial glands and basement membrane cells appeared to undergo mitosis, cell basal membranes had vacuoles, the ratio of ciliated and non-ciliated tissue decreased and the endometrium appeared to have inadequate secretion, which was not conducive to embryo implantation, resulting in infertility or recurrent miscarriage (13). Fainaru et al (14) observed that MHC II are capable of supporting angiogenesis and promoting the growth of EM lesions. Protein spot no. 83 was similar to that of eukaryotic initiation factor (EIF)-3. The protein spot with the same difference as spot no. 26 (immunoglobulin heavy chain variable region) was not reported to correlate with endometrial EM; therefore, further investigation is required.

Spot no. 55 in Uyghur uterine EM serum was similar to the actin-like-7- $\alpha$ and $\gamma$-aminobutyric-acid receptor subunit. The former is a cytoskeletal protein involved in cell division, movement and signaling, and the latter may adjust the transmission of signals between nerve cells and inhibit 
neurons. It has been reported (15) that the $\pi$ subunits of the $\gamma$-aminobutyric acid receptor are more abundant in the womb and may alter the sensitivity of the receptor to endogenous steroid hormones. Sadeghi et al (16) observed that HOXA10 was capable of regulating the expression of the endometrial GABAA receptor and EM. Its isoelectric point and molecular weight were particularly similar to those of cyclin A1. Cyclin A1 is involved in the regulation of G1/S and G2/M phase conversion and therefore correlates closely with DNA replication and cell division. Spot no. 123 was similar to that of hepatocyte-derived growth factor (HDGF) and the 14-3-3 protein family members. HDGF is one of the members of growth factors, with a function of promoting growth of liver cells, fibroblasts, smooth muscle cells and endothelial cells. The expression of HDGF in numerous cancer patients is significantly greater than that in healthy controls, indicating that HDGF correlates with tumor cell growth and angiogenesis. The 14-3-3 protein is widely distributed in eukaryotic cells and through binding to effector molecules it induces their phosphorylation, which is important in cellular signal transduction. In the diagnosis of cortex striatum spinal degeneration and subacute spongiform encephalopathy, the 14-3-3 protein is likely to be an important element. Ametzazurra et al (17) observed that the differential expression of the 14-3-3 protein in signal transduction may be a marker for EM. Zhang et al (18) demonstrated that the 14-3-3 protein was lost in Han normal endometrial cells. Certain findings are similar to those of differentially expressed proteins in Xinjiang Uygur EM patients.

The results of the study by Zhang et al (18) demonstrated that EM may be a class of multifactorial diseases, similar to diabetes, asthma and cancer. The preliminary view is that EM exhibits ethnic differences in protein expression. However, the specific mechanisms for these proteins during the pathogenesis of EM remains unclear and requires further investigation. The identified proteins are expected to become specific molecular markers and next step is to apply these to develop a non-traumatic EM diagnostic test. This diagnostic test should have ideal sensitivity, specificity, with a satisfactory positive and negative prediction value and be cheap and simple to obtain. Furthermore, according to the study of tumor-directed therapy, nonspecific tumor antigens and antibody heterogeneity markedly impact the development of orientated therapy. Therefore, initially markers for EM must be located prior to creating an EM-orientated treatment. With the progression and development of proteomics research, numerous techniques may be applied to investigate EM. The expression profiling of screened related proteins with high throughput, sensitivity and accuracy is of great significance in order to verify the molecular mechanisms underlying the occurrence and development of EM, and to identify early diagnostic markers and therapeutic targets.

\section{References}

1. Valle RF and Sciarra JJ: Endometriosis: treatment strategies. Ann NY Acad Sci 997: 229-239, 2003.

2. Dunn SR and Pennington MJ: Proteomics: From Protein Sequence to Function. BIOS Scientific Publishers, Oxford, UK, pp122-134, 2001.

3. Rout MP, Aitchison JD, Suprapto A, Hjertaas K, Zhao Y and Chait BT: The yeast nuclear pore complex: composition, architecture, and transport mechanism. J Cell Biol 148: 635-651, 2000.

4. Newman JR, Wolf E and Kim PS: A computationally directed screen identifying interacting coiled coils from Saccharomyces cerevisiae. Proc Natl Acad Sci USA 97: 13203-13208, 2000.

5. Wall DB, Kachman MT, Gong SS, Parus SJ, Long MW and Lubman DM: Isoelectric focusing nonporous silica reversed-phase high-performance liquid chromatography/electrospray ionization time-of-flight mass spectrometry: a three-dimensional liquid-phase protein separation method as applied to the human erythroleukemia cell-line. Rapid Commun Mass Spectrom 15: 1649-1661, 2001

6. Wang $\mathrm{H}$ and Hanash S: Multi-dimensional liquid phase based separations in proteomics. J Chromatogr B Analyt Technol Biomed Life Sci 787: 11-18, 2003.

7. Nisolle M, Casanas-Roux F and Donnez J: Immunohistochemical analysis of proliferative activity and steroid receptor expression in peritoneal and ovarian endometriosis. Fertil Steril 68: 912-919, 1997.

8. Küster B, Mortensen P, Andersen JS and Mann M: Mass spectrometry allows direct indetification of protein in large genomes. Protemics 1: 641-650, 2001

9. Demir AY, Groothuis PG, Nap AW, Punyadeera C, de Goeij AF, Evers JL and Dunselman GA: Menstrual effluent induces epithelial-mesenchymal transitions in mesothelial cells. Hum Reprod 19: 21-29, 2004.

10. Miettinen M and Fetsch JF: Distribution of keratins in normal endothelial cells and a spectrum of vascular tumors: implications in tumor diagnosis. Hum Pathol 31: 1062-1067, 2000.

11. Gao YH, Qi NL, Zhang P, et al: A novel human gene spindlin 1, encoding a protein localized in the cell nucleus and inducing NIH3T3 cell's transformation. Prog Nat Sci 14: 1058-1062, 2004 (In Chinese).

12. Cody CW, Prasher DC, Westler WM, Prendergast FG and Ward WW: Chemical structure of the hexapeptide chromophore of the Aequorea green-fluorescent protein. Biochemistry 32: 1212-1218, 1993

13. Clup P, Nüsslein-Volhard C and Hopkins N: High-frequency germ-line transmission of plasmid DNA sequences injected into fertilized zebrafish eggs. Proc Natl Acad Sci USA 88: 7953-7957, 1991.

14. Fainaru O, Adini A, Benny O, et al: Dendritic cells support angiogenesis and promote lesion growth in a murine model of endometriosis. FASEB J 22: 522-529, 2008.

15. Braun DP, Ding J, Shen J, Rana N, Fernandez B and Dmowski WP: Relationship between apoptosis and the number of macrophages in eutopic endometrium from women with and without endometriosis. Fertil Steril 78: 830-835, 2002.

16. Sadeghi $\mathrm{H}$ and Taylor HS: HOXA10 regulates endometrial GABAA $\pi$ receptor expression and membrane translocation. Am J Physiol Endocrinol Metab 298: E889-E893, 2010.

17. Ametzazurra A, Matorras R, García-Velasco JA, et al: Endometrial fluid is a specific and non-invasive biological sample for protein biomarker identification in endometriosis. Hum Reprod 24: 954-965, 2009.

18. Zhang H, Niu Y, Feng J, Guo H, Ye X and Cui H: Use of proteomic analysis of endometriosis to identify different protein expression in patients with endometriosis versus normal controls. Fertil Steril 86: 274-282, 2006. 